\title{
Percepção do usuário quanto aos atributos de engajamento: uma análise qualitativa
}

\author{
Igor Sampaio $^{1}$, Leonardo Vasconcelos ${ }^{1}$, José Viterbo ${ }^{1}$, Daniela Trevisan ${ }^{1}$ \\ ${ }^{1}$ Instituto de Computação - Universidade Federal Fluminense (UFF) \\ 24.210-240 - Niterói - RJ - Brazil \\ igorgarcia@id.uff.br, \{lvasconcelos, viterbo, daniela\}@ic.uff.br
}

\begin{abstract}
Today, user motivations go beyond merely completing an operational task in information systems. Solutions must also be designed to promote user engagement. Engagement can be defined as the quality of user experience. Because engagement is more tied to the behavioral and subjective part, there is a need to capture and measure affective responses during interactions. Consequently, the evolution of the measurement methods of such experiences becomes paramount in order to assess the subjective aspects intrinsic to the attributes of engagement to systematically meet this new demand. This study will approach a qualitative analysis of the characteristics of engagement, aiming to discover the importance of each one from the user's perspective and the correlation between them. Given this scenario, we aim to contribute to an assessment from the user's perception of what factors effectively involve them during their experience of using the systems. Our results presented the attributes of engagement most valued by users and which have a dependency relationship between them.
\end{abstract}

Resumo. Atualmente, as motivações do usuário vão além de meramente completar uma tarefa operacional nos sistemas de informação. As soluções devem também ser projetadas para promover o engajamento dos usuários. O engajamento pode ser definido como uma qualidade de experiência do usuário. Pelo engajamento estar mais atrelado à parte comportamental e subjetiva, surge a necessidade de capturar e mensurar respostas afetivas durante as interações. Consequentemente, torna-se primordial a evolução dos métodos de medição de tais experiências, a fim de aferir os aspectos subjetivos intrínsecos aos atributos de engajamento para sistemicamente atender a essa nova demanda. Este estudo abordará uma forma de análise qualitativa das características do engajamento objetivando descobrir a importância de cada uma sob a ótica do usuário e a correlação entre elas. Diante deste cenário, visamos contribuir com uma avaliação a partir da percepção do usuário, a respeito de quais fatores efetivamente os envolvem durante sua experiência de uso com os sistemas. Nossos resultados apresentaram os atributos de engajamento mais valorizados pelos usuários e quais possuem uma relação de dependência entre eles.

\section{Introdução}

Atualmente, as motivações do usuário vão além de meramente completar uma tarefa operacional nos sistemas de informação. As soluções devem também ser projetadas para promover o engajamento dos usuários [O'Brien and Toms 2008]. O engajamento pode 
ser definido como um estado psicológico onde o indivíduo se propõe a investir integralmente o seu esforço para solucionar uma tarefa [Liang et al. 2018].

O'Brien and Toms [O'Brien and Toms 2008] definem o engajamento como uma qualidade de experiência do usuário e, por ele estar mais atrelado à parte comportamental e subjetiva, surge a necessidade de capturar e mensurar respostas afetivas durante as interações. Consequentemente, torna-se primordial a evolução dos métodos de medição de tais experiências, a fim de aferir os aspectos subjetivos intrínsecos aos atributos de engajamento para sistemicamente atender a essa nova demanda.

O estudo [O'Brien and Cairns 2015] considera os questionários como sendo um instrumento apropriado para coleta de dados qualitativos que suportem este tipo de medição e respectivas análises. Em linha com esta transformação, este estudo abordará uma forma de análise qualitativa das características do engajamento [O'Brien and Toms 2008], objetivando descobrir a importância de cada uma sob a ótica do usuário e a correlação existente entre elas [Mukaka 2012].

Diante deste cenário, este estudo visa contribuir com uma avaliação a partir da percepção do usuário, a respeito de quais fatores efetivamente os envolvem durante sua experiência de uso com os sistemas. Demonstrou-se, qualitativamente, quais atributos de engajamento são mais valorizados e qual grau de intensidade da relação de dependência existente entre eles. A partir da generalização de tais resultados é possível enriquecer os designs de novas aplicações com foco nas expectativas subjetivas e legítimas dos usuários.

Este artigo está dividido em sete seções, incluindo esta. Na Seção 2, descrevemos no referencial teórico todos os conceitos que serão usados em nosso estudo. Na seção 3 , levantamos os trabalhos relacionados presentes na literatura. Na Seção 4, descrevemos nossa metodologia. Na seção 5, interpretamos nossos resultados. Na Seção 6, apresentamos as nossas conclusões. E, para finalizar, na Seção 7 apresentamos os trabalhos futuros.

\section{Referencial Teórico}

Neste estudo é importante identificar os conceitos de engajamento do usuário, como os seus atributos estão divididos, a forma em que os seus processos estão organizados e como mensurá-lo. Além disso, é fundamental entender o coeficiente de Pearson para a análise dos resultados.

\subsection{Engajamento do Usuário}

O termo engajamento é definido de diferentes maneiras na literatura. Liang et al. [Liang et al. 2018] define o engajamento como um estado psicológico onde o indivíduo se propõe a investir integralmente o seu esforço para solucionar uma tarefa. Ksiazek et al. [Ksiazek et al. 2016] apresenta o engajamento como um conjunto amplo de fatores que apresentam todos os tipos de envolvimento e atenção do usuário. Em [Attfield et al. 2011], o engajamento do usuário é uma qualidade da experiência que enfatiza os aspectos positivos da interação - em particular, o fato de ser cativado pela tecnologia. Já a sigla UE - User Engagement - é um padrão bastante usado para o tema.

Neste artigo, utilizaremos a extensa revisão bibliográfica apresentada em [O'Brien and Toms 2008] e os estudos de [Attfield et al. 2011] como referência conceitual para nosso estudo. Em [O'Brien and Toms 2008], a definição de engajamento consiste em uma qualidade de experiência do usuário caracterizada por atributos, tais como: 
apelo estético e sensorial, atenção, desafio, durabilidade, feedback do sistema, interatividade, controle percebido pelo usuário, prazer e variedade/novidade.

\subsubsection{Atributos de engajamento}

De acordo com [O'Brien and Toms 2008], o referencial teórico que informa o engajamento do usuário integra atributos do sistema (feedback, desafio, apelo estético e sensorial) com as percepções do usuário quanto ao sistema (controle, prazer, atenção). Assim, o entendimento do engajamento é conceitualmente um trabalho holístico para desvendar a integração das variáveis do sistema e do usuário e a combinação entre elas a fim de ampliar os limites da experiência do usuário: de meramente superficial para memorável e prazerosa. Para tal, tais variáveis ou características do sistema e do usuário foram definidas como atributos de engajamento, tais como [O'Brien and Toms 2008], [Lalmas et al. 2014] e [Attfield et al. 2011]: estética, atenção focada, desafio, durabilidade, feedback, interatividade, controle, prazer, apelo sensorial e variedade/novidade.

A Estética (EA) se refere à aparência visual atraente da interface do sistema. A Atenção focada (AT) é concentração seletiva do usuário no uso do sistema. O Desafio (DE) trata fluidez e facilidade de navegação com níveis adequados de desafio. A Durabilidade (DU) é a frequência de uso e tempo de permanência no sistema. O Feedback (FE) é resposta satisfatória do sistema para o usuário. A Interatividade (IN) é o grau adequado de troca de informações entre sistema e usuário durante interação. O Controle (CT) é o nível de controle do sistema percebido pelo usuário na interação o Prazer (PR) é o afeto positivo, satisfação percebida pelo usuário durante a interação. O Apelo sensorial (AS) é a interface desperta os sentidos do usuário de forma inteligente. A Variedade/novidade (VN) é a interface surpreender o usuário pela diversidade ou novidade.

Segundo [O'Brien and Toms 2008] e [Lalmas et al. 2014], tais atributos estão presentes nas diferentes etapas do processo de engajamento. A intensidade de cada atributo pode variar de acordo com a etapa do processo, o tipo de interação com sistema, a natureza da tecnologia adotada, as expectativas do usuário e sua experiência com a tecnologia.

\subsubsection{Processo de engajamento}

O processo de engajamento consiste em quatro estágios: um ponto de engajamento inicial, um período de engajamento sustentado, desengajamento e (possivelmente) reengajamento. O resultado obtido em [O'Brien and Toms 2008] é a identificação dos atributos que caracterizam cada um dos quatro estágios. O ponto de engajamento é iniciado pelo apelo estético ou pela nova apresentação da interface, pelas motivações e interesses do usuário e pela habilidade e desejo dos usuários de se situarem na interação e perceberem que há tempo suficiente para usar o aplicativo. Já no engajamento, é sustentado quando os usuários conseguem manter sua atenção e interesse na aplicação e é caracterizado por emoções positivas. Os usuários desejam personalizar a interface para atender às suas necessidades e receber feedback adequado e oportuno do aplicativo. Além disso, eles querem perder a percepção do tempo e dos outros durante as interações, mas manter a consciência dos outros nos casos em que o envolvimento é reforçado pela interação social. 
No desengajamento, os usuários se desligam do aplicativo por vários motivos, como a usabilidade da tecnologia, distrações ou fatores externos. Este estágio pode resultar em emoções positivas ou negativas. Após um desengajamento, o usuário pode retornar ao sistema, sendo esta fase chamada de reengajamento. Os usuários podem percorrer as etapas de engajamento várias vezes durante uma única sessão, logo o reengajamento é intrínseco ao modelo. Este estágio assume as mesmas características do ponto de engajamento. Vale ressaltar que, o engajamento nem sempre faz parte das experiências dos usuários com os aplicativos, por diferentes motivos (ex.: preferência por compras em uma loja física, por aulas presenciais, falta de tempo ou a multitarefa) que impediram seu envolvimento [O'Brien and Toms 2008].

\subsubsection{Medição dos atributos de engajamento}

Com intuito de validar a relevância dos atributos de engajamento constados em [O'Brien and Toms 2008], [Lalmas et al. 2014] e [Attfield et al. 2011] para sistemas de informação, este estudo fundamentou a escolha do método de medição mais adequado, baseado em [O'Brien and Cairns 2015]. Consta que os questionários possibilitam capturar os aspectos subjetivos das experiências do usuário com os sistemas, coletando fatores experimentais individuais dessa interação. Como por exemplo: a motivação do usuário por um sistema específico ou a sua resposta emocional diante de seu uso.

Em [O'Brien and Cairns 2015], apesar da capacidade de avaliar o envolvimento dos usuários com uma visão mais abrangente e holística, o questionário deve contemplar as muitas dimensões do UE [O'Brien and Toms 2008], deve demonstrar confiabilidade, validade e capacidade de generalização. Em [Lalmas et al. 2014], tal generalização é vista como um facilitador que, através da padronização, viabiliza comparações entre diferentes naturezas de sistemas, diferentes grupos de usuários, etc. Em adição, o trabalho [Attfield et al. 2011] afirma que questionários e escalas bem desenhados e empiricamente validados permitem que os resultados sejam explorados, definidos e padronizados.

No estudo [O'Brien and Cairns 2015], é feita a análise de validade e de confiabilidade de um tipo de questionário e de escala específicos, denominado de User Engagement Scale (UES), para ambientes de navegação on-line de notícias. Criado originalmente para área de multimídia educacional e já aplicado em diversas áreas sistêmicas, o UES já obteve resultados positivos quanto à sua validade e confiabilidade em algumas áreas de aplicação. Esta escala captura de forma confiável as dimensões do envolvimento do usuário [O'Brien and Cairns 2015], também citadas em [O’Brien and Toms 2008], denominando-as de subescalas de UES. As suas medidas ajudam a entender a percepção dos usuários sobre as interações sistêmicas a partir de dados comportamentais. Com este objetivo, utilizamos alguns conceitos de UES como referência para preparação do questionário do nosso estudo.

\subsection{Coeficiente de Pearson}

Dentro da estatística, o coeficiente de correlação releva a existência e o nível de relacionamento entre duas variáveis. Esta correlação pode ser positiva, neutra ou negativa e pode assumir diferentes níveis de intensidade de muito alta/forte até muito baixa/fraca. Para enriquecer a análise de dados de uma pesquisa, vale estudar a correlação entre todas as 
variáveis, fixando uma variável independente e avaliando sua relação com a outra variável que a princípio é dependente da primeira. O coeficiente é calculado da seguinte forma:

$$
r=\frac{\sum_{i=1}^{n}\left(x_{i}-\bar{x}\right)\left(y_{i}-\bar{y}\right)}{\sum_{i=1}^{n} \sqrt{\left(x_{i}-\bar{x}\right)^{2} \cdot \sqrt{\left(y_{i}-\bar{y}\right)^{2}}}}=\frac{\operatorname{cov}(X, Y)}{\sqrt{\operatorname{var}(X) \cdot \operatorname{var}(Y)}}
$$

Na matriz de correlação, a diagonal terá sempre o resultado 1 pois representa a correlação e uma variável com ela mesma. O coeficiente de Pearson (r) varia de -1 a 1 , notando que -1 é uma perfeita correlação negativa (existe correlação inversamente proporcional entre as duas variáveis) e 1 é uma perfeita correlação positiva (existe correlação diretamente proporcional entre as duas variáveis). Um coeficiente de correlação próximo de zero indica que não há relação entre as duas variáveis.

A regra de interpretação do coeficiente de correlação é funciona da seguinte maneira [Mukaka 2012] : r $>0.9$ para mais ou para menos indica uma correlação muito forte. $0.7<\mathrm{r}<0.9$ positivo ou negativo indica uma correlação forte. $0.5<\mathrm{r}<0.7$ positivo ou negativo indica uma correlação moderada. $0.3<\mathrm{r}<0.5$ positivo ou negativo indica uma correlação fraca. $0<\mathrm{r}<0.3$ positivo ou negativo indica uma correlação desprezível.

\section{Trabalhos Relacionados}

Há muitos estudos recentes que investigaram os fatores envolvidos na adoção dos usuários, intenção de uso e aceitação de aplicações. No entanto, não existem muitos estudos que se focam na percepção do usuário quanto à relevância dos atributos de engajamento, revelada durante sua interação com os sistemas de informação.

Attfield et al. [Attfield et al. 2011], propõem uma abordagem para relacionar e desenvolver medidas unificadas de engajamento do usuário, visando contribuir com novas perspectivas para definir e explicá-las. Lalmas et al. [Lalmas et al. 2014], apresentam diversas formas de medição do engajamento, relatando vantagens, desvantagens e restrições de cada uma.

Mukaka [Mukaka 2012], apresenta o conceito e a forma de utilização do coeficiente de correlação de Pearson para o domínio de pesquisas médicas. O’Brien and Toms [O'Brien and Toms 2008], realizam uma extensa e crítica revisão bibliográfica multidisciplinar acompanhada de um estudo exploratório dos usuários. Em seu trabalho eles definiram dez atributos de engajamento de usuários e os estágios do processo de engajamento.

Vasconcelos et al. [Vasconcelos et al. 2019] verificaram como comentários negativos - ou reclamações -, relacionados a fatores que influenciam diretamente o engajamento, influenciam a avaliação dos usuários sobre aplicativos de mobile crowdsourcing. Os aplicativos foram avaliados por cinco características que influenciam o engajamento, sendo elas: usabilidade, durabilidade, controle, confiança e eficiência. Os resultados experimentais verificaram que a hipótese é verdadeira para o fator de durabilidade.

O'Brien and Cairns [O'Brien and Cairns 2015] demonstram um questionário específico, o User Engagement Scale (UES), e as suas respectivas subescalas no domínio de notícias online. Os autores analizaram o potencial do UES para medir corretamente as experiências de informações humanas. Os autores acreditam que o User Engagement 
Scale consegue em vários contextos extrair confiavelmente as dimensões do engajamento do usuário..

\section{Metodologia}

Nossa metodologia foi dividida em quatro fases: (i) selecionar um perfil de usuários, (ii) coletar dados, (iii) analisar os dados e (iv) interpretar os resultados. Na fase (i), selecionamos estudantes de cursos de computação da Universidade Federal Fluminense. Eles foram selecionados pelo fato de sua formação oferecer um amplo domínio tecnológico, um alto grau de atualização tecnológica, e pouca disponibilidade de tempo. Trinta e nove estudantes se disponibilizaram a participar da pesquisa, no entanto, dois estudantes não autorizaram o uso de seus dados para fins de pesquisa de forma pública. Sendo assim, eles foram retirados da análise dos resultados.

No final, o estudo foi realizado com trinta e sete participantes. 89,2\% dos participantes possuíam idade entre 19 e 39 anos, 8,1\% dos participantes possuíam idade entre 40 e 59 anos, 5,4\% possuíam idade menor que 18 anos ou maior que 50 anos. Todos os participantes possuem a expertise do tema de estudo, sendo 17 participantes graduados e 20 participantes pós-graduados correspondendo à $45,9 \%$ e $54,1 \%$ do percentual total respectivamente. Logo, todos eles possuem o conhecimento adequado para agregar valor à pesquisa de forma consciente e pragmática.

$\mathrm{Na}$ fase (ii), os dados foram coletados através da aplicação de um questionário online. Escolhemos essa opção pelo objetivo do artigo ser validar a relevância dos atributos de engajamento para sistemas de informação. O questionário foi composto por 13 questões, distribuídas de forma a reunir dados de consentimento, demográficos e engajamento. A primeira pergunta se referia ao consentimento ou autorização do pesquisado quanto ao uso dos resultados exclusivamente para fins de pesquisa de forma pública. Essa questão foi levantada para a pesquisa estar em linha com as exigências do Conselho de Ética. As duas perguntas seguintes solicitavam a faixa etária e nível de escolaridade dos participantes. As dez perguntas restantes, presentes no Apêndice A, estavam associadas a apenas um atributo de engajamento (UE), seguindo os conceitos de UES com as devidas adaptações.

As respostas para as perguntas relacionadas aos atributos do engajamento utilizaram a escala de Likert com cinco pontos no intervalo (de "discordo totalmente" que equivale ao número 1 a "concordo totalmente" que equivale ao número 5). Além disso, foi disponibilizado um espaço para comentários em cada questão. Os comentários não eram campos obrigatórios para que as pessoas com pouca disponibilidade de tempo ou interesse não se desmotivassem a respondê-lo. Após a definição da estrutura do questionário no Google Forms, ele foi enviado aos participantes e divulgado através de redes sociais. Nenhum tipo de remuneração foi oferecido, o prazo de resposta foi de cinco dias úteis. Ao término do prazo, o próprio Google Forms bloqueou o questionário para respostas. As fases (iii) e (iv) são apresentadas nas próximas seções.

\section{Interpretação dos Resultados}

Nesta seção, primeiramente apresentamos o diagnóstico dos resultados de nosso estudo comparados a abordagem do User Engagement Escale apresentado 
em [O’Brien and Cairns 2015]. Depois, apresentamos a correlação dos atributos utilizando o coeficiente de Pearson.

\subsection{Diagnóstico dos resultados}

Foi necessário elaborar perguntas vinculadas diretamente aos atributos aqui citados, para que os participantes expressassem sua reação à cada um dos componentes de sua experiência com a aplicação. Tendo definido o envolvimento do usuário e elaborado algumas de suas principais características, os resultados do questionário estão apresentados na Tabela 1 .

Tabela 1. Resultados

\begin{tabular}{|c|c|c|c|c|c|c|c|c|c|c|}
\hline Sigla & $\mathbf{1}$ & $\mathbf{2}$ & $\mathbf{3}$ & $\mathbf{4}$ & $\mathbf{5}$ & $\mathbf{\% 1}$ & $\mathbf{\% 2}$ & $\mathbf{\% 3}$ & $\mathbf{\% 4}$ & $\mathbf{\% 5}$ \\
\hline IN & 34 & 3 & 0 & 0 & 0 & $91,9 \%$ & $8,1 \%$ & $0,0 \%$ & $0,0 \%$ & $0,0 \%$ \\
\hline EA & 17 & 17 & 3 & 0 & 0 & $45,9 \%$ & $45,9 \%$ & $8,1 \%$ & $0,0 \%$ & $0,0 \%$ \\
\hline AT & 2 & 12 & 16 & 7 & 0 & $5,4 \%$ & $32,4 \%$ & $43,2 \%$ & $18,9 \%$ & $0,0 \%$ \\
\hline DU & 17 & 11 & 4 & 3 & 2 & $45,9 \%$ & $29,7 \%$ & $10,8 \%$ & $8,1 \%$ & $5,4 \%$ \\
\hline CT & 18 & 16 & 3 & 0 & 0 & $48,6 \%$ & $43,2 \%$ & $8,1 \%$ & $0,0 \%$ & $0,0 \%$ \\
\hline DE & 35 & 1 & 0 & 1 & 0 & $94,6 \%$ & $2,7 \%$ & $0,0 \%$ & $2,7 \%$ & $0,0 \%$ \\
\hline PR & 13 & 13 & 7 & 4 & 0 & $35,1 \%$ & $35,1 \%$ & $18,9 \%$ & $10,8 \%$ & $0,0 \%$ \\
\hline AS & 6 & 15 & 12 & 3 & 1 & $16,2 \%$ & $40,5 \%$ & $32,4 \%$ & $8,1 \%$ & $2,7 \%$ \\
\hline FE & 33 & 2 & 2 & 0 & 0 & $89,2 \%$ & $5,4 \%$ & $5,4 \%$ & $0,0 \%$ & $0,0 \%$ \\
\hline VN & 13 & 16 & 5 & 3 & 0 & $35,1 \%$ & $43,2 \%$ & $13,5 \%$ & $8,1 \%$ & $0,0 \%$ \\
\hline
\end{tabular}

Nossa abordagem diferenciou-se do UES original, exigindo sua adaptação para atender a análise do engajamento do usuário à soluções sistêmicas de forma genérica e abrangente. Além disso, foi necessário elaborar afirmativas que realmente motivassem os participantes do estudo a se engajarem com o questionário.

Quanto a aparência visual da interface do sistema, o usuário crê que uma interface amigável e esteticamente atraente influi em seu engajamento (45.9\% concordam totalmente e $45.9 \%$ concordam parcialmente). Já em concentração durante o uso da aplicação, nem todos os usuários concordam que uma aplicação que promova a não percepção da passagem do tempo é sinônimo de engajamento, já que 43,2\% são indiferentes a afirmação e outros $24.3 \%$ discordam.

No que se diz respeito a interação do usuário com a aplicação, é quase unânime (94.6\%) que a mesma seja fácil, simples e fluída durante seu uso. Acerca da relevância do sistema motivar o usuário a utilizá-lo e a compartilhá-lo, os usuários se mostram divididos: $45.9 \%$ concordam totalmente, $29.7 \%$ concordam parcialmente, $10.8 \%$ são indiferentes, $8.1 \%$ discordam parcialmente e $5.4 \%$ discordam totalmente. Portanto, fica difícil concluir algo a respeito da relação entre a frequência de uso e a motivação provocada pelo sistema.

Em relação a confiança e credibilidade da aplicação, a grande maioria (89.2\%) acredita que a aplicação tem o dever de passar segurança a seus usuários. Quanto ao grau adequado de troca de informações entre o sistema e o usuário, 91,9\% marcaram a opção "concordo totalmente", o que se leva a crer que os usuários acham importante a execução de tarefas sem qualquer dificuldade, de forma simples e fluída. 
No tocante a controle percebido, $48.6 \%$ concorda totalmente e $43.2 \%$ concorda parcialmente que é relevante o usuário perceber que está no controle da aplicação, com completo domínio. Com referência ao prazer, os respondentes também se mostram bem divididos. Um sistema que proporciona uma experiência satisfatória e gratificante não necessariamente é um sistema engajador, uma vez que $35.1 \%$ concordam totalmente, $35.1 \%$ concordam parcialmente , $18.9 \%$ são indiferentes e $10.8 \%$ discordam parcialmente.

Audição, fala, visão e toque dividem também os usuários, ou seja, é difícil dizer a partir desta afirmativa, que uma aplicação que desperta os sentidos com uso de apelos sensoriais, necessariamente engaja o usuário final. Com relação as recomendações sugeridas pelo aplicativo, o público novamente fica dividido. $35.1 \%$ concorda totalmente, $43.2 \%$ concorda parcialmente , $13.5 \%$ é indiferente e $8.1 \%$ discorda parcialmente. Com as respostas obtidas, não há assertividade para afirmar que um sistema que forneça sugestões aderentes às preferências do usuário é engajador. Notou-se que o fato de sugerir novidades e surpreender é visto de forma positiva por alguns pesquisados.

\subsection{Correlação de atributos}

Após a obtenção das respostas do questionário, se fez necessário o cálculo do coeficiente de Pearson a fim de definir a correlação entre os atributos, como pode ser visto na Tabela 2 . Interatividade, Desafio e Feedback possuem uma alta correlação, 0.997. Isto diz que atributos que envolvam facilidade, fluidez e simplicidade possuirão execuções de tarefas sem dificuldade, além de credibilizar a experiência do usuário final. Estética e Controle também possuem o mais alto grau de correlação obtido neste estudo ( $r=0.997)$, o que leva a crer que uma aparência visual atraente leva a um controle percebido pelo usuário durante as interações com a aplicação.

Tabela 2. Matriz de Correlação de Pearson

\begin{tabular}{|c|c|c|c|c|c|c|c|c|c|c|c|}
\hline Sigla & Sub Escala (UES) & IN & EA & AT & DU & CT & DE & PR & AS & FE & VN \\
\hline IN & Interatividade & 1 & 0,672 & $-0,425$ & 0,879 & 0,729 & 0,997 & 0,611 & $-0,070$ & 0,997 & 0,532 \\
\hline EA & Estética / Aparência & 0,672 & 1 & 0,051 & 0,942 & 0,997 & 0,619 & 0,941 & 0,567 & 0,644 & 0,972 \\
\hline AT & Atenção / Concentração & $-0,425$ & 0,051 & 1 & $-0,133$ & 0,009 & $-0,447$ & 0,317 & 0,825 & $-0,395$ & 0,248 \\
\hline DU & Durabilidade & 0,879 & 0,942 & $-0,133$ & 1 & 0,965 & 0,845 & 0,891 & 0,342 & 0,860 & 0,870 \\
\hline CT & Controle & 0,729 & 0,997 & 0,009 & 0,965 & 1 & 0,679 & 0,938 & 0,522 & 0,703 & 0,957 \\
\hline DE & Desafio & 0,997 & 0,619 & $-0,447$ & 0,845 & 0,679 & 1 & 0,565 & $-0,125$ & 0,997 & 0,476 \\
\hline PR & Prazer & 0,611 & 0,941 & 0,317 & 0,891 & 0,938 & 0,565 & 1 & 0,705 & 0,599 & 0,971 \\
\hline AS & Apelo Sensorial & $-0,070$ & 0,567 & 0,825 & 0,342 & 0,522 & $-0,125$ & 0,705 & 1 & 0,064 & 0,700 \\
\hline FE & Feedback & 0,997 & 0,644 & $-0,395$ & 0,860 & 0,703 & 0,997 & 0,599 & $-0,064$ & 1 & 0,505 \\
\hline VN & Variedade / Novidade & 0,532 & 0,972 & 0,248 & 0,870 & 0,957 & 0,476 & 0,971 & 0,700 & 0,505 & 1 \\
\hline
\end{tabular}

Com fator de correlação igual a 0.971, Prazer está altamente correlacionado a Variedade/Novidade, isto é, uma aplicação que surpreende seu usuário através da diversidade e da novidade, proporcionará satisfação. Durabilidade, Estética possui fator de correlação de 0.942 , o que é considerado alto. Portanto, a frequência de uso do sistema está altamente correlacionada a interagir com uma interface visualmente atraente. Controle e Durabilidade também estão correlacionados $(r=0.965)$, o que pode apontar que quanto mais o usuário utiliza a aplicação, mais ele se percebe no controle dela. Prazer e Controle também estão altamente correlacionados. Com 0.938 de coeficiente, uma experiência prazerosa está ligada à alta percepção de controle do sistema por parte do usuário.

Variedade e Controle também se correlacionam, $r=0.957$. Portanto, sistemas em que as recomendações sugeridas estão de acordo com as preferências do usuário, 
os fazem ter a percepção que possuem controle sobre a aplicação. Prazer e Estética possuem coeficiente de 0.941 , ou seja, aplicações com interface amigável com apelo visual, geram satisfação durante seu uso. Variedade e Estética se correlacionam com grau de 0.972, isto é, interfaces visualmente atrentes, usualmente surpreende o usuário. Atenção/Concentração e Apelo Sensorial não obtiveram grau significativo de correlação com nenhum outro atributo.

Questionários são comumente usados para medir atitudes em relação a sistemas e percepções de experiências de pesquisa. Embora a validade aparente de tais medidas tenha sido estabelecida através do uso repetido em pesquisa de recuperação de informação, sua confiabilidade e validade mais ampla normalmente não são examinadas. Tal fato ameaça a validade interna. A avaliação do auto-relato dos questionários é importante não só para a validade interna dos estudos e, por extensão, aumento da confiança nos resultados, mas também para examinar as construções de interesse ao longo do tempo e entre diferentes domínios e sistemas [O’Brien and Cairns 2015].

\section{Conclusão}

Este trabalho avalia a percepção dos usuários a respeito de quais aspectos de fato engajam os mesmos durante o momento da interação com um sistema de informação. Neste sentido, conclui-se que as características de natureza subjetiva, presentes nos sistemas, ganharam de fato maior importância sob a ótica dos usuários. A eficiência anteriormente exigida das aplicações, meras executoras de tarefas, não é mais suficiente para envolver esta nova geração de usuários, inseridos nas redes sociais e ávidos por estímulos e inovação.

Em linha com este contexto atual, este estudo demonstrou qualitativamente, através do uso de um questionário, quais atributos de engajamento [O'Brien and Toms 2008] são mais valorizados pelos usuários e quais possuem uma relação de dependência entre eles. Estes resultados podem agregar valor às pesquisas quantitativas de cunho técnico que reportam taxas de downloads, taxas de acesso, notas de resenha, etc. Mediante o uso de coeficiente de correlação Pearson, como métrica estatística, o trabalho revelou o grau de relacionamento e de intensidade entre tais atributos, valorizando uma análise objetiva e pragmática associada às percepções subjetivas. Em adição, o estudo utilizou as definições de UES presentes em [O'Brien and Cairns 2015] e generalizou sua aplicação para além do âmbito de notícias online, para soluções sistêmicas em geral sem domínio específico.

\section{Trabalhos futuros}

Apesar do questionário ser uma ferramenta adequada para coletar dados de forma qualitativa, a homogeneidade e o tamanho reduzido do grupo de participantes da pesquisa não permitem que os resultados sejam extrapolados para um universo mais abrangente. Torna-se necessário, expandir o público de forma a se obter respostas mais significativas e maior aleatoriedade ao processo, através de maior diversidade do perfil dos participantes.

Outra possibilidade é aumentar o número de questões por atributo de engajamento e fomentar a escrita dos comentários no questionário de forma a ter mais riqueza de dados qualitativos a serem analisados. É de extrema importância que se faça uso dos 
parâmetros de validação estatística. E por fim, segundo [O'Brien and Cairns 2015], existem evidências que sugerem que o UES pode-se diferenciar entre sistemas e condições experimentais. Portanto, é recomendável aplicar este estudo à um domínio em específico, com o interesse em testar essas evidências.

\section{Referências}

Attfield, S., Kazai, G., Lalmas, M., and Piwowarski, B. (2011). Towards a science of user engagement (position paper). In WSDM workshop on user modelling for Web applications, pages 9-12.

Ksiazek, T. B., Peer, L., and Lessard, K. (2016). User engagement with online news: Conceptualizing interactivity and exploring the relationship between online news videos and user comments. New media \& society, 18(3):502-520.

Lalmas, M., O’Brien, H., and Yom-Tov, E. (2014). Measuring user engagement. Synthesis Lectures on Information Concepts, Retrieval, and Services, 6(4):1-132.

Liang, H., Wang, M.-M., Wang, J.-J., and Xue, Y. (2018). How intrinsic motivation and extrinsic incentives affect task effort in crowdsourcing contests: A mediated moderation model. Computers in Human Behavior, 81:168-176.

Mukaka, M. M. (2012). A guide to appropriate use of correlation coefficient in medical research. Malawi Medical Journal, 24(3):69-71.

O'Brien, H. L. and Toms, E. G. (2008). What is user engagement? a conceptual framework for defining user engagement with technology. Journal of the American society for Information Science and Technology, 59(6):938-955.

O'Brien, H. and Cairns, P. (2015). An empirical evaluation of the user engagement scale (ues) in online news environments. Information Processing \& Management, 51(4):413-427.

Vasconcelos, L., Barcellos, R., Viterbo, J., Bernardini, F., Boscarioli, C., and Nunes, E. (2019). How factors that influence engagement impact users' evaluations in mobile app stores. In International Conference on Human-Computer Interaction, pages 571-584. Springer.

\section{A. Perguntas do questionário}

As perguntas foram formuladas para cada um dos atributos de engajamento, definidos na seção 2.1. As alternativas de resposta refletiam a escala de Likert com cinco pontos de intervalo:

1. Discordo totalmente

2. Discordo parcialmente

3. Indiferente

4. Concordo parcialmente

5. Concordo totalmente

O questionário utilizado no trabalho e os comentários acrescentados podem ser encontrados em:

https://github.com/igorgbs/waihcws_19_questions 